\title{
Letter to Editor: Lower Urinary Tract Mesh Perforations Following the Insertion of Mid-urethral Tapes or Transvaginal Mesh: A Tertiary Unit's Experience - Saidan, et al. 2019
}

\section{Wael Agur}

University Hospital Crosshouse, NHS Ayrshire \& Arran, United Kingdom

*Corresponding author: Wael Agur, NHS Ayrshire \& Arran, University Hospital Crosshouse, Kilmarnock KA2 OBE, United Kingdom, Tel: +44-(0)-1563-825-383, Fax: +44-(0)-1563-825-490

\section{Dear Editor in Chief}

We read the paper from Dr. Saidan and colleagues [1] with interest. Mesh removal surgery is a highly important and emerging field that requires sharing of the learning experiences from centres worldwide.

It appears all women who had urinary tract perforation following mesh tape in the study underwent partial (rather than total) removal surgery, including the 8 women whose devices had been in place for less than 8 weeks. It is relatively easy to totally remove a device that had been recently implanted, before scar tissue is matures and removal becomes difficult. It is not clear whether separate groin incisions were employed during surgery to achieve total removal of the women's transobturator mesh devices.

Authors stated they had thought that three women had total removal of their prolapse mesh devices, however, one presented later with recurrent bladder erosion that required further excision surgery. Such recurrence suggests that the original surgical removal was only partial, not total. Have any of the 40 women given consent for photographs of their explanted mesh materials to be taken for comparison of size/length to the original implant? Published photographs of the explants would be quite useful for readers of papers reporting on vaginal mesh removal surgery.

For both incontinence and prolapse devices, it was not clear whether the authors had provided an information leaflet or a patient decision form to facilitate counselling and to help the 40 women balance the benefits and risks of a total versus a partial mesh removal surgery.

Total removal of a mesh device that had been previously partially removed is believed to be quite difficult. In the circumstance that some women subsequently require total removal of their residual mesh device, what preoperative imaging and subsequent surgical approach would the authors consider and/ or recommend?

The authors state that patients presented with urinary tract perforations 'at a later date, rather than intra-operative injury'. However, 18 (45\%) women presented less than a year of mesh implantation, of whom $8(45 \%)$ presented less than 8 weeks of the original surgery. Such relatively soon presentation of almost half of the study population suggests an unrecognised intra-operative trocar injury, rather than a late mesh complication with its different pathophysiology and natural history.

The 3-month follow-up period is too short to conclude that partial mesh removal surgery was successful. Are authors planning a longer term follow up study of this cohort? There are reports [2] of a number of Scottish women who had felt initial improvement following removal surgery, however, subsequently reported feeling worse or much worse on the longer term. Such outcome appears to be more common in women who underwent partial, rather than total, removal surgery. 
The Methodology Section describes 'patient-reported outcomes'; however, the Results section appears to show only unvalidated physician-reported impressions. Did the authors consider the widely-used Patient Global Impression for Improvement (PGI-I) [3]? It is a validated patient-reported tool and would have provided an accurate measurement of the outcomes of mesh removal surgery. Similarly, the authors reported the results of unvalidated classification of severity of complication rather than a validated one. A good example here would be the widely used Dindo-Clavien System [4].

Currently, there is no formal process of accreditation by UK Royal Colleges for mesh removal centres. Therefore, it is perhaps best to describe the service as 'self-recognised', rather than 'accredited'.

Finally, the risk incidence of urinary tract perforation with mesh procedures appears to be more frequent than the authors' description of 'rare'. As the number of first single vaginal mesh procedures performed in Scotland is around 20,000 [5], the 40 women reported in the study suggest a risk incidence of $0.2 \%$ (1:500). Such risk incidence is described by NHS England [6] as 'uncommon' (1:100 to 1:1000 1 person in a village), not 'rare' (1:1000 to $1: 10000 \sim 1$ person in a small town). Even with a conservative risk estimate, the real-life incidence would almost certainly be higher than $0.2 \%$ - as there must have been women with perforations who had been treated outside the study and/or elsewhere in Scotland outside the authors' hospital.
The authors are to be congratulated for publishing their series of mesh removal surgery. It is highly important to share the various experiences and to help standardise the approach in the emerging field of management of mesh complications.

\section{References}

1. Saidan D, Tyagi V, Granitsiotis P, Guerrero K (2019) Lower urinary tract mesh perforations following the insertion of mid-urethral tapes or transvaginal mesh: A tertiary unit's experience. Int Arch Urol Complic 5: 1-6.

2. (2019) Management of pelvic mesh complications in Scotland - preliminary results of a service evaluation co-designed by patients and clinicians. The Scottish Parliament.

3. Yalcin I, Bump RC (2003) Validation of two global impression questionnaires for incontinence. Am J Obstet Gynecol 189: 98-101.

4. Dindo D, Demartines N, Clavien PA (2004) Classification of surgical complications: A new proposal with evaluation in a cohort of 6336 patients and results of a survey. Ann Surg 240: 205-213.

5. Morling JR, McAllister DA, Agur W, Fischbacher CM, Glazener CM, et al. (2017) Adverse events after first, single, mesh and non-mesh surgical procedures for stress urinary incontinence and pelvic organ prolapse in Scotland, 19972016: A population-based cohort study. Lancet 389: 629640.

6. (2017) Synthetic vaginal mesh tape procedure for the surgical treatment of stress urinary incontinence in women. Patient Information Leaflet. NHS England: 9-16. 